Case Report

\title{
Mitral Valve Replacement with a Mechanical Valve for Severe Mitral Regurgitation in a Small Dog
}

\author{
Daisuke Taguchi, ${ }^{1,2}$ Isamu Kanemoto, ${ }^{1}$ Satoko Yokoyama, ${ }^{1,3}$ \\ Masashi Mizuno, ${ }^{1,4}$ and Makoto Washizu ${ }^{5}$ \\ ${ }^{1}$ Chayagasaka Animal Hospital, 1-1-5 Shinnishi, Chikusa, Nagoya, Aichi 464-0003, Japan \\ ${ }^{2}$ Green Animal Hospital, 179 Tamakake-maeda, Nanbu, Sannohe, Aomori 039-0101, Japan \\ ${ }^{3}$ Miyashita Animal Hospital, 5-8-29 Keigoya, Kure, Hiroshima 737-0012, Japan \\ ${ }^{4}$ Veterinary Internal Medicine of Nihon University, 1866 Kameino, Fujisawa, Kanagawa 252-8510, Japan \\ ${ }^{5}$ Animal Teaching Hospital of Gihu University, 1-1 Yanagido, Gifu 501-1193, Japan \\ Correspondence should be addressed to Isamu Kanemoto; kanemoto@ta2.so-net.ne.jp
}

Received 16 April 2014; Revised 7 July 2014; Accepted 14 July 2014; Published 6 August 2014

Academic Editor: Paola Roccabianca

Copyright (C) 2014 Daisuke Taguchi et al. This is an open access article distributed under the Creative Commons Attribution License, which permits unrestricted use, distribution, and reproduction in any medium, provided the original work is properly cited.

\begin{abstract}
A seven-year-old Shih Tzu with refractory repeated pulmonary edema and syncope was presented for surgical operation. From the results of cardiovascular examinations, the dog was diagnosed as severe mitral regurgitation (ACVIM consensus class D) and mild tricuspid regurgitation. The dog first underwent surgery with mitral valve plasty; however, the results were unsatisfactory due to severe damage of the whole mitral valve. The operation was quickly changed to mitral valve replacement using a mechanical valve $(19 \mathrm{~mm})$. The dog survived surgery and lived for 2 years and one month after operation using long-term anticoagulant (warfarin) therapy in spite of several thrombosis-related events.
\end{abstract}

\section{Introduction}

Mitral regurgitation (MR) caused by degenerative valve disease is quite common in small breed dogs [1]. There are two methods of surgical operation for MR [2, 3]: mitral valvuloplasty (MVP) which is the repair of the valve itself and mitral valve replacement (MVR) which entails an exchange with an artificial valve, either mechanical or bioprosthetic. Although MVR using a mechanical heart valve is excellent in its durability, it needs to be maintained with lifetime anticoagulant therapy because of the foreign body response. In contrast, although MVR using a bioprosthetic valve does not require long-term anticoagulant therapy, it is less durable $[2,3]$.

There are a few clinical case reports of MVR using a mechanical valve [4-6], and there are some clinical $[7,8]$ and experimental [9] reports of MVR using a bioprosthetic valve in medium-size or large breed dogs. However, there is only one clinical case report of MVR using a mechanical valve in a small breed dog and of the postoperative long-term result of anticoagulant therapy in such dogs [6].

This report presents the first successful case of MVR using a mechanical valve in a small breed dog, which survived 2 years and one month after operation using longterm anticoagulant (warfarin) therapy in spite of several thrombosis-related events.

\section{Case Presentation}

The dog was a male Shih Tzu, 7 years old, weighing $5.3 \mathrm{~kg}$. It was diagnosed with MR and treatment had begun 1 year 3 months earlier. Dyspnea occurred 2 months before the first visit and then recently recurred every few days. Syncope occurred 1 month and 8 days before the first visit. Although the dog's prognosis was considered to be very poor, he was presented to our hospital for surgery at the owner's strong wish. On physical examination, auscultation revealed Levine 3-4/6 systolic murmur (SM) at the apex, 2/6 SM at 


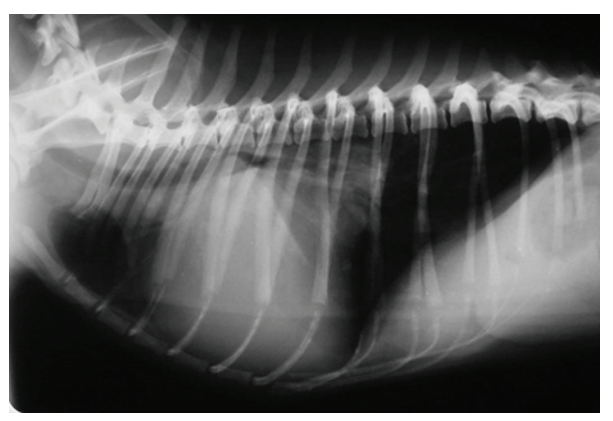

(a)

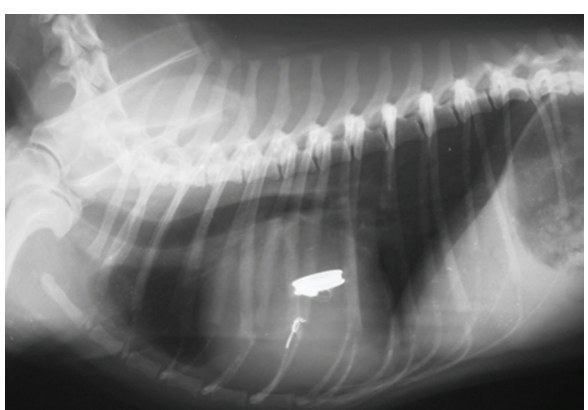

(b)

Figure 1: (a) Preoperative and (b) postoperative L-R X-ray photographs. Preoperative VHS $12.0 \mathrm{v}$ decreased to $10.1 \mathrm{v} 3.5$ months after operation, and a mechanical valve is recognized in (b).

the tricuspid valve area, and crackle in the lung area. On chest $\mathrm{X}$-ray examination, elevation of trachea and enlargement of cardiac size (VHS $12.0 \mathrm{v}$, CTR $89 \%$ ), especially in the left atrium and ventricle, were recognized (Figure 1(a)).

Echocardiogram revealed dilation of left atrium (LA/Ao: 3), enlargement of mitral valve ring size (diameter $28.4 \mathrm{~mm}$ ), prolapsed chordae of the anterior leaflet, and ruptured chorda of the posterior one. Color Doppler showed severe mitral regurgitation from the posterior commissure to the posterior side of the left atrium. Fractional shortening (FS) showed a normal range $(35-45 \%)$ which means the presence of impaired myocardial cell contraction. The left ventricular end diastolic internal dimension (LVIDd) distended remarkably $49.5 \mathrm{~mm}$, and mild tricuspid regurgitation was recognized. Severe (ACVIM consensus class D) MR with mild TR was diagnosed. Although the prognosis was considered poor, open heart surgery was performed as a last measure.

Open Heart Assist Method. This entails surface-cooling hypothermia (sHT) combined with low-flow cardiopulmonary bypass $(\mathrm{CPB})[10]$. In this case, the lowest esophageal temperature (ET) was $20.1^{\circ} \mathrm{C}$, the aortic cross-clamp (ACC) time was 79 minutes, the lowest pump flow was $60 \mathrm{~mL} / \mathrm{kg} /$ minute, and the recorded CPB time was 2 hours and 53 minutes. Anesthesia: the dog was administered acepromazine $(0.05 \mathrm{mg} / \mathrm{kg} \mathrm{SC})$, atropine $(0.025 \mathrm{mg} / \mathrm{kg} \mathrm{SC})$, and hydroxyzine $(1 \mathrm{mg} / \mathrm{kg} \mathrm{SC})$ at 30 minutes and 15 minutes before induction, respectively. Anesthesia was induced with thiamylal $(12.5 \mathrm{mg} / \mathrm{kg} \mathrm{IV})$ and maintained with inhalation of isoflurane in oxygen and intermittent administration of pancuronium $(0.06 \mathrm{mg} / \mathrm{kg} \mathrm{IV})$. After administration of heparin (400 IU/kg IV), a $12 \mathrm{Fr}$ venous cannula was inserted from the left jugular vein into the right atrium. A $2.0 \mathrm{~mm}$ diameter metal arterial cannula was then inserted through the left carotid artery toward the heart and connected with the CPB circuit (NAPS III and Mera S-circuit, Senko Ika Kogyo). Deep hypothermia was slowly induced by surface-cooling with ice bags. At ET $30^{\circ} \mathrm{C}$, the left 5 th intercostal space was opened and the surgical field was distended by cutting the costal cartilage of the 6 th rib. The pericardium was opened by cutting parallel under the left phrenic nerve and sutured to the chest wall to create a pericardial cradle. An aortic root cannula for cardioplegia was inserted in the aortic root. A two-stage (18-24 F) venous tube was additionally inserted from the right appendage into the posterior vena cava and connected with $\mathrm{CPB}$. At ET $25^{\circ} \mathrm{C}, \mathrm{CPB}$ was started. After both the ascending aorta and main pulmonary artery were crossclamped together, cold $\left(4^{\circ} \mathrm{C}\right)$ cardioplegia solution $(10 / \mathrm{mL} / \mathrm{kg}$ St Thomas II solution) was immediately injected antegrade through an aortic root cannula. The left atrium was cut transversely above the coronary groove. The whole mitral valve was hypertrophied with mucous degeneration, and many portions of the anterior and posterior leaflets prolapsed widely like a yacht sail, and one chorda of the posterior leaflet ruptured near the posterior commissure.

Surgical Method. At first, MVP was performed. The prolapsed anterior leaflet and ruptured posterior leaflet were sutured with the triangular plication (McGoon) method [11] and the posterior commissural valve ring was sutured with the mattress (JH Kay) method [12], as we reported previously [13]. However, the leak test showed a great volume of regurgitation. Therefore, the MVR method was performed instead of MVP. After the whole mitral valve with chordae tendineae was cut off leaving the base of the valve annulus, a $19 \mathrm{~mm}$ diameter Bjork-Shiley oblique valve for aortic valve was attached there conversely with three 5-0 prolines and the continuous suture method (Figure 2(a)). After the left atrium was closed and intracardiac air removed completely, the aortic cross-clamp (ACC) was removed. Immediately, a spontaneous beat occurred from ventricular fibrillation with one DC shock. The patient was weaned from CPB 40 minutes after removing the ACC, and also from controlled ventilation 15 hours after operation.

Postoperative Course. Anticoagulant therapy using warfarin was started on the second day after surgery. Appetite and vigor recovered on the 4 th day after operation, and the patient was discharged 12 days after surgery. Since then, warfarin therapy was controlled by the attending doctor. On 3.5-month postoperative chest X-ray examination, VHS decreased from preoperative $12.0 \mathrm{v}$ to $10.1 \mathrm{v}$ (Figure $1(\mathrm{~b})$ ). The echocardiogram revealed disappearance of MR, reduced LVIDd from preoperative $49.5 \mathrm{~mm}$ to $32.6 \mathrm{~mm}$, and slightly persisting TR. 


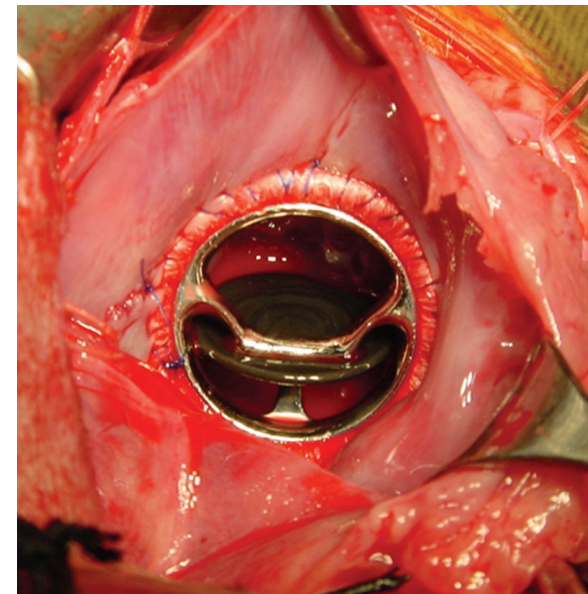

(a)

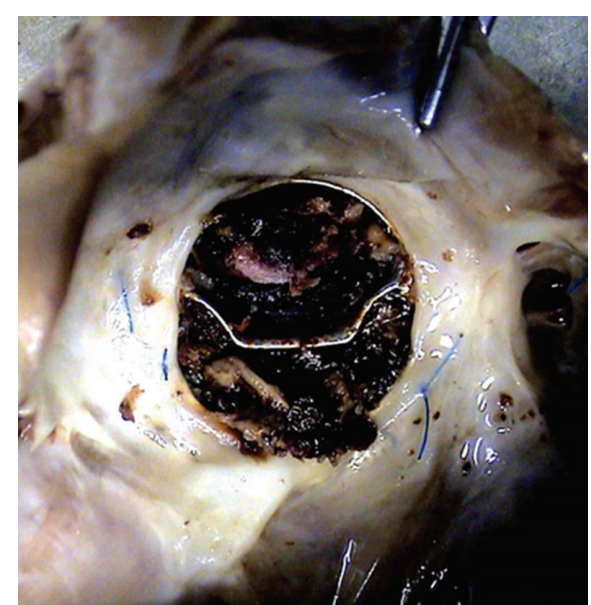

(b)

FIGURE 2: (a) An intraoperative photograph shows Bjork-Shiley oblique valve for aortic valve attached conversely at the mitral valve position. (b) An autopsy photograph (surgeon view) shows the artificial valve covered with a great amount of thrombi.

After discharge from our hospital, warfarin was administered at $0.25-0.55 \mathrm{mg} / \mathrm{kg} /$ day (almost $0.35 \mathrm{mg} / \mathrm{kg} /$ day) by the attending doctor. Six events associated with the artificial valve reported by the attending doctor and owner were a swelling of the right leg and bleeding from the nail root 3.5 months after operation, mild paralysis of the left leg 8 months after operation, and mild seizure at 1 year, 1 year 9 months, and 2 years after operation, nearly all of which seemed to be associated with blood clot formation except for bleeding from the nail root. Two years after operation, prosthetic valve dysfunction due to thrombus was suspected because the opening and closing sounds of the artificial valve became suddenly inaudible. After warfarin administration was increased, the opening and closing sounds of the artificial valve were again auscultated. One month after the event, however, the dog had an abrupt onset of severe coughing and dyspnea during the night, although he acted normally during daytime. On auscultation, sounds of the artificial valve were not audible, but severe lung rales were heard in both lung fields. The dog was finally euthanized because of symptomatic worsening as time advanced, despite various rescue treatments (Figure 3).

Autopsy Findings. The artificial valve was covered with a large amount of thrombi (Figure 2(b)). Trachea was filled with a large amount of pink bubbly fluid.

\section{Discussion}

In human [2,3] and veterinary medicine [13-17], MVP is currently preferred over MVR for providing good QOL. However, it is difficult to completely repair severely damaged valves with MVP in a terminal stage case. In human medicine, there are many reports related to MVR [2, 3]. However, in veterinary literature, to our knowledge, there are only three reports of MVR using a mechanical valve in clinical cases which were operated in medium-size to large breed dog
[4-6]. There is only one reported case of surgery with MVR using a mechanical valve $(19 \mathrm{~mm})$ in a small $\operatorname{dog}(4.3 \mathrm{~kg})$ [6], but the dog died during surgery due to oversized valve prosthesis. In our small dog $(5.3 \mathrm{~kg})$, we first attempted to use MVP [13] (McGoon [11] combined with the JH Kay [12] method), but the results were unsatisfactory due to severe damage of the entire mitral valve. Therefore, we quickly switched from MVP to MVR with a mechanical valve (19 $\mathrm{mm}$ diameter for aortic valve; the smallest available valve prosthesis). The dog survived surgery and after discharge remained alive for 2 years.

In this case, sHT was combined with CPB. Deep sHT can be easily performed in a small dog, and CPB can assist the systemic circulation during ACC and rewarming using a heat-exchanger. $\mathrm{CPB}$ using peripheral vessels improves cardiac access in small dogs, although HT is needed because of low-flow $\mathrm{CPB}$ due to decreased venous return volume. A combination of deep sHT with low-flow $\mathrm{CPB}$ enhances the benefits and minimizes the disadvantages of both techniques in small dogs [10].

Regarding postoperative anticoagulant therapy, there is only one report [6] from 3 clinical reports [4-6] of MVR using a mechanical valve in dogs. Orton and colleagues [6] reported administration of warfarin at the same dosage in 6 dogs as in the guideline for humans; but all died of thrombosis-related causes, 5 dogs within one year and 1 dog at 5.25 years (median survival after surgery: 4.5 months). The same warfarin dosage as used in the guideline for humans is recommended to maintain the prothrombin time-based INR of 2.5-3.5 [2, 3, 18]. According to an experimental study using Hall-Haster mitral valves [19], warfarin dosage of a thrombotest aimed at $20-25 \%$ normal coagulation activity was ineffective in preventing thrombosis, and a more intensive antithrombotic prophylaxis was required.

In the case under study, the warfarin dosage was $0.25-$ $0.55 \mathrm{mg} / \mathrm{kg} /$ day, but mainly $0.35 \mathrm{mg} / \mathrm{kg} /$ day was administered. However, 6 thrombosis-related events occurred in spite 


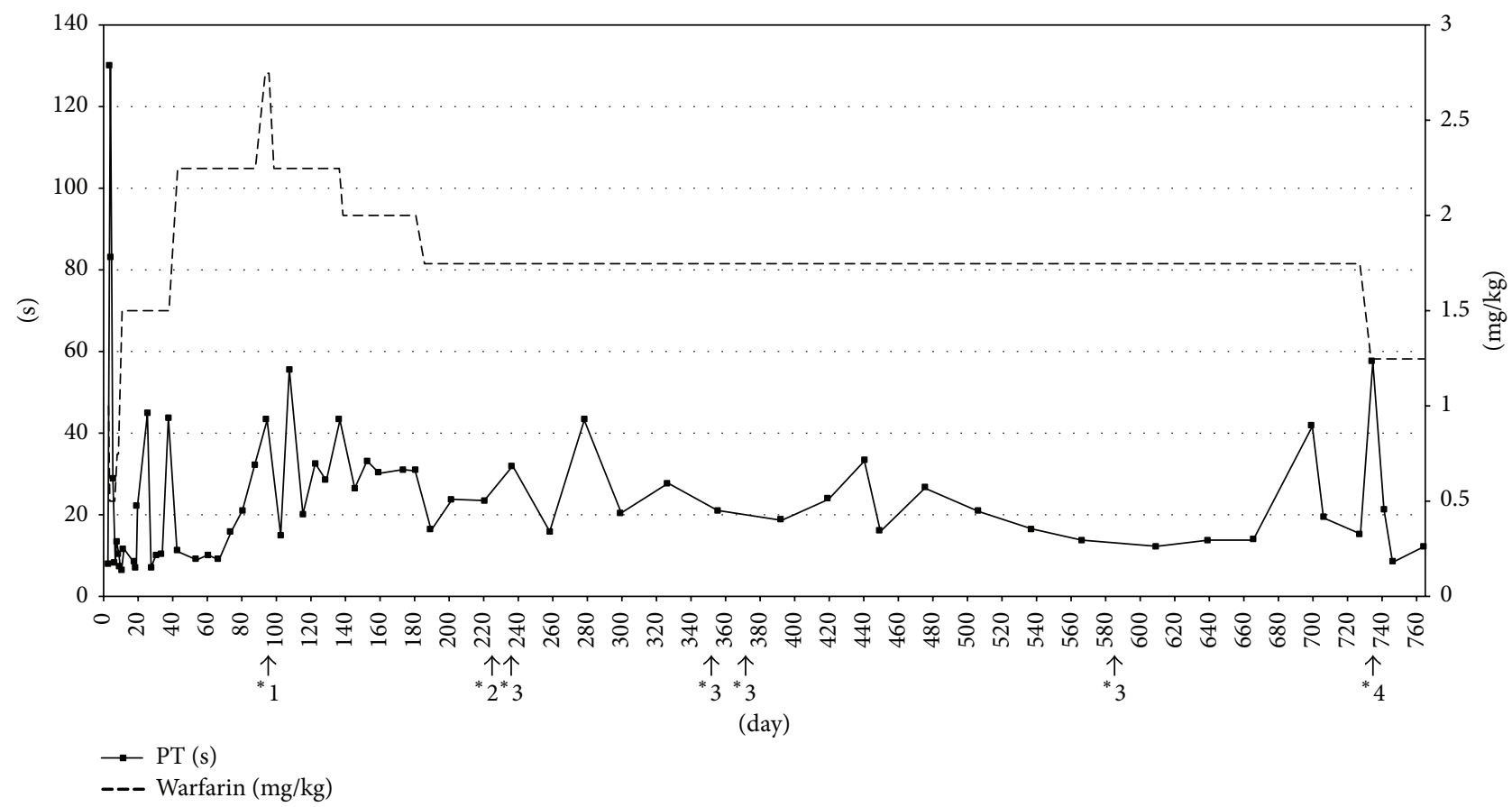

FIGURE 3: Changes of prothrombin time (PT) and dosage of warfarin administered after operation. * 1 swelling of the right leg and bleeding from the nail root (95 days after operation). ${ }^{*} 2$ mild paralysis of left leg and mild seizure (226 days after operation). ${ }^{*} 3$ mild seizure (229, 348 , 370,585 days after operation). ${ }^{*} 4$ prosthetic valve dysfunction due to thrombus (733 days after operation).

of prothrombin time (PT) $7.5-24 \mathrm{sec}$ or $<24 \mathrm{sec}$, except for swelling of the right leg and bleeding from the nail root 3.5 months after operation; the PT underwent great changes in spite of the same warfarin dosage. Unfortunately, the dog died of prosthetic valve thrombosis 2 years and one month after operation. From our experience with this case, it seemed that oral administration of warfarin only was not suitable for controlling the prothrombin time in a dog, unlike in a human. Klement and colleagues [20] reported MVR using the same Bjork-Shiley Monostat valve in large experimental dogs for which anticoagulation was started 48 hours postoperatively by giving warfarin and adjusted on a daily basis to maintain the prothrombin time at 1.5 to 2 times the preoperative value. However, wide variations of prothrombin time were controlled by adding aspirin and dipyridamole to warfarin. Unfortunately, a follow-up survey was not conducted until 3 weeks after operation. Still another problem with dogs is that the normal prothrombin time varies widely $(6-10 \mathrm{sec}$ [21], 9-14 sec [22], and 14-18 sec [23]) at each facility. Thus, each facility should have its own prothrombin time or use the prothrombin time-based INR.

Recently, new anticoagulant drugs (Purazakisa, Izagureru, Erikyusu) have become available, and in the future, their effects are expected to serve well in the anticoagulant therapy for MVR in dogs.

\section{Conclusions}

We experienced the first successful case of MVR using a mechanical valve in a small breed dog, which survived 2 years and one month after operation using long-term anticoagulant (warfarin) therapy in spite of several thrombosis-related events. Anticoagulant therapy using only warfarin makes it very difficult to control PT for MVR in dog.

\section{Conflict of Interests}

The authors declare that there is no conflict of interests regarding the publication of this paper.

\section{Acknowledgment}

This paper was presented at the 13th Federation Asian Veterinary Association (FAVA) Congress, October 25-28, 2004, in Seoul, Korea, and as a case in the paper in Open Heart Surgery with Deep Hypothermia and Cardiopulmonary Bypass in Small and Toy Dogs, Vet. Surg., 39: 674-679, 2010.

\section{References}

[1] M. D. Kittleson and R. D. Kienle, "Myxomatous atrioventricular valvular degeneration," in Small Animal Cardiovascular Medicine, M. D. Kittleson and R. D. Kienle, Eds., pp. 297-318, Mosby, Kimberton, Pa, USA, 1998.

[2] R. P. Gallegos, T. Gudbjartsson, and S. Aranki, "Mitral valve replacement," in Cardiac Surgery in the Adult, L. H. Cohn, Ed., pp. 849-876, McGraw-Hill Medical, New York, NY, USA, 4th edition, 2012.

[3] N. T. Kouchoukos, E. H. Blackstone, F. L. Hanley, and J. K. Kirklin, "Mitral valve disease," in Kirklin/Barratt-Boyes Cardiac Surgery, N. T. Kouchoukos, E. H. Blackstone, F. L. Hanley, and 
J. K. Kirklin, Eds., pp. 484-536, Elsevier Saunders, Philadelphia, $\mathrm{Pa}$, USA, 4th edition, 2013.

[4] G. E. Eyster, W. Weber, S. Chi et al., "Mitral valve prosthesis for correction of mitral regurgitation in a dog," Journal of the American Veterinary Medical Association, vol. 168, no. 12, pp. $1115-1118,1976$.

[5] E. M. Breznock, "Tricuspid and mitral valvular disease: valve replacement," Seminars in Veterinary Medicine and Surgery (Small Animal), vol. 9, no. 4, pp. 234-239, 1994.

[6] E. C. Orton, T. B. Hackett, K. Mama, and J. A. Boon, "Technique and outcome of mitral valve replacement in dogs," Journal of the American Veterinary Medical Association, vol. 226, no. 9, pp. 1508-1511, 2005.

[7] R. N. White, R. L. Stepien, R. A. Hammond et al., "Mitral valve replacement for the treatment of congenital mitral dysplasia in a bull terrier," The Journal of Small Animal Practice, vol. 36, no. 9, pp. 407-410, 1995.

[8] L. Behr, V. Chetboul, C. C. Sampedrano et al., "Beating heart mitral valve replacement with a bovine pericardial bioprosthesis for treatment of mitral valve dysplasia in a bull terrier," Veterinary Surgery, vol. 36, no. 3, pp. 190-198, 2007.

[9] K. Takashima, A. Soda, R. Tanaka, and Y. Yamane, "Long-term clinical evaluation of mitral valve replacement with porcine bioprosthetic valves in dogs," Journal of Veterinary Medical Science, vol. 70, no. 3, pp. 279-283, 2008.

[10] I. Kanemoto, D. Taguchi, S. Yokoyama, M. Mizuno, H. Suzuki, and T. Kanamoto, "Open heart surgery with deep hypothermia and cardiopulmonary bypass in small and toy dogs," Veterinary Surgery, vol. 39, no. 6, pp. 674-679, 2010.

[11] D. C. McGoon, "Repair of mitral insufficiency due to ruptured chordae tendineae," Journal of Thoracic Cardiovascular Surgery, vol. 39, pp. 357-362, 1960.

[12] J. H. Kay and W. S. Egerton, "The repair of mitral insufficiency associated with ruptured chordae tendineae," Annals of Surgery, vol. 157, no. 3, pp. 351-360, 1963.

[13] I. Kanemoto, S. Shibata, H. Noguchi, S. Chimura, M. Kobayashi, and Y. Shimizu, "Successful mitral valvuloplasty for mitral regurgitation in a dog," Japanese Journal of Veterinary Science, vol. 52, no. 2, pp. 411-414, 1990.

[14] L. S. Boggs, S. J. Dewan, and S. E. Ballard, "Mitral valve reconstruction in a toy-breed dog," Journal of the American Veterinary Medical Association, vol. 209, no. 11, pp. 1872-1876, 1996.

[15] I. Kanemoto, H. Suzuki, D. Taguchi, S. Yokoyama, M. Mizuno, and T. Kanamoto, "Successful surgical repair for severe mitral regurgitation in five small-breed dogs," Veterinary Surgery, vol. 33, no. 5, p. 435-E12, 2004.

[16] L. G. Griffiths, E. C. Orton, and J. A. Boon, "Evaluation of techniques and outcomes of mitral valve repair in dogs," Journal of the American Veterinary Medical Association, vol. 224, no. 12, pp. 1941-1945, 2004.

[17] M. Uechi, "Mitral valve repair in dogs," Journal of Veterinary Cardiology, vol. 14, no. 1, pp. 185-192, 2012.

[18] P. D. Stein, J. S. Alpert, J. E. Dalen, D. Horstkotte, and A. G. G. Turpie, "Antithrombotic therapy in patients with mechanical and biological prosthetic heart valves," Chest, vol. 114, no. 5, pp. 602S-610S, 1998.

[19] J. Dale, A. O. Aasen, F. Resch, B. Semb, K. Stadskleiv, and P. Lilleaasen, "Mitral disc valve implantation in the dog: early and late valve thrombosis and its prevention," European Surgical Research, vol. 15, no. 5, pp. 249-255, 1983.
[20] P. Klement, C. M. Feindel, H. E. Scully et al., "Mitral valve replacement in dogs. Surgical technique and postoperative management," Veterinary Surgery, vol. 16, no. 3, pp. 231-237, 1987.

[21] Kirk and Bistner's Handbook of Veterinary Procedures and Emergency Treatment, p. 888, WB Saunders, Philadelphia, Pa, USA, 7th edition, 2000.

[22] R. M. Jacobs, J. H. Lumsden, and J. A. Taylor, "Canine and feline reference values," in Kirk's Current Veterinary Therapy XIII, Small Animal Practice, p. 1221, W. B. Saunder, Philadelphia, $\mathrm{Pa}$, USA, 2000.

[23] M. B. Brooks, "Coagulation disease," in Saunders Manual of Small Animal Practice, S. J. Birchard and R. G. Sherding, Eds., pp. 256-264, Saunders Elsevier, St. Louis, Mo, USA, 3rd edition, 2006. 

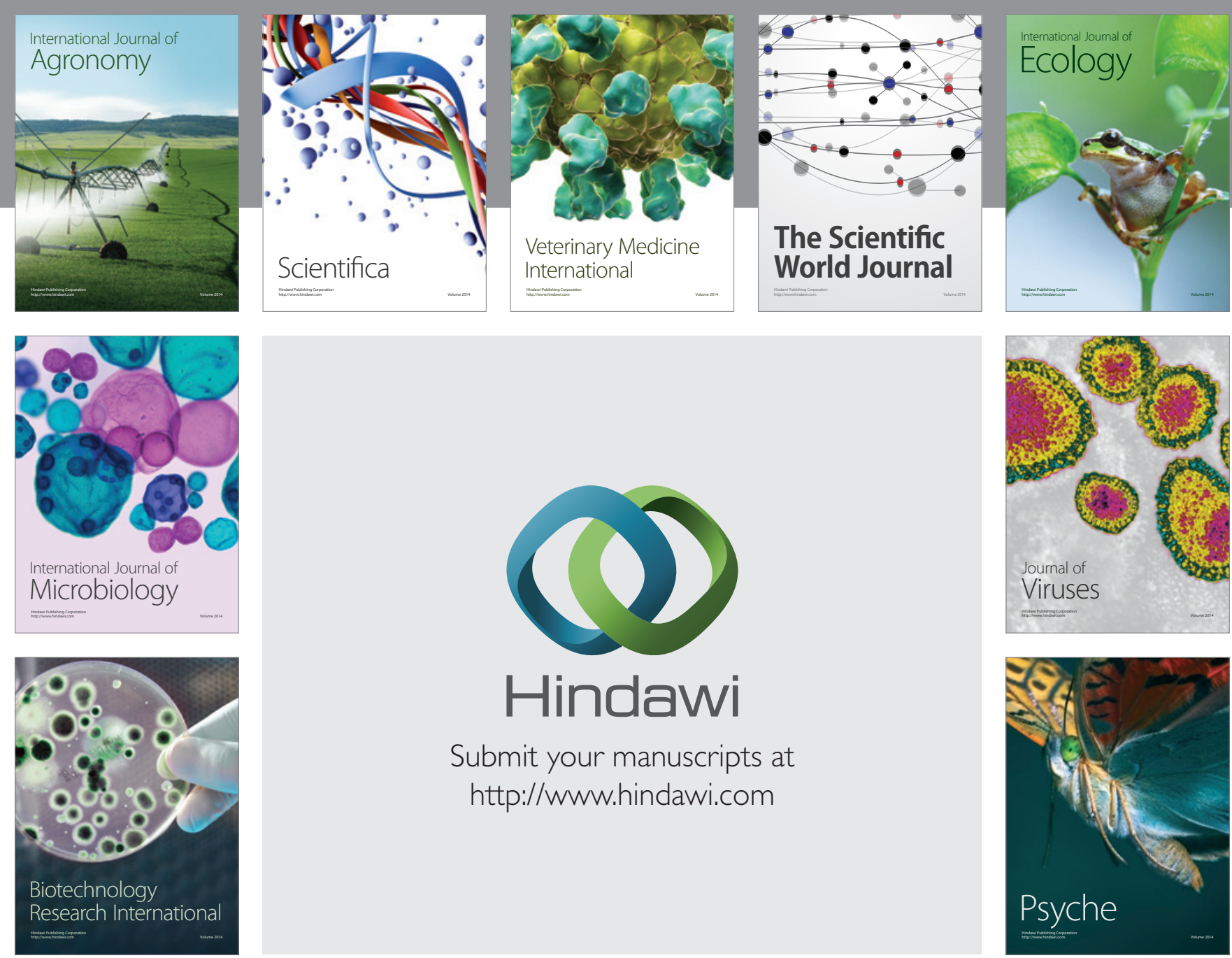

Submit your manuscripts at http://www.hindawi.com
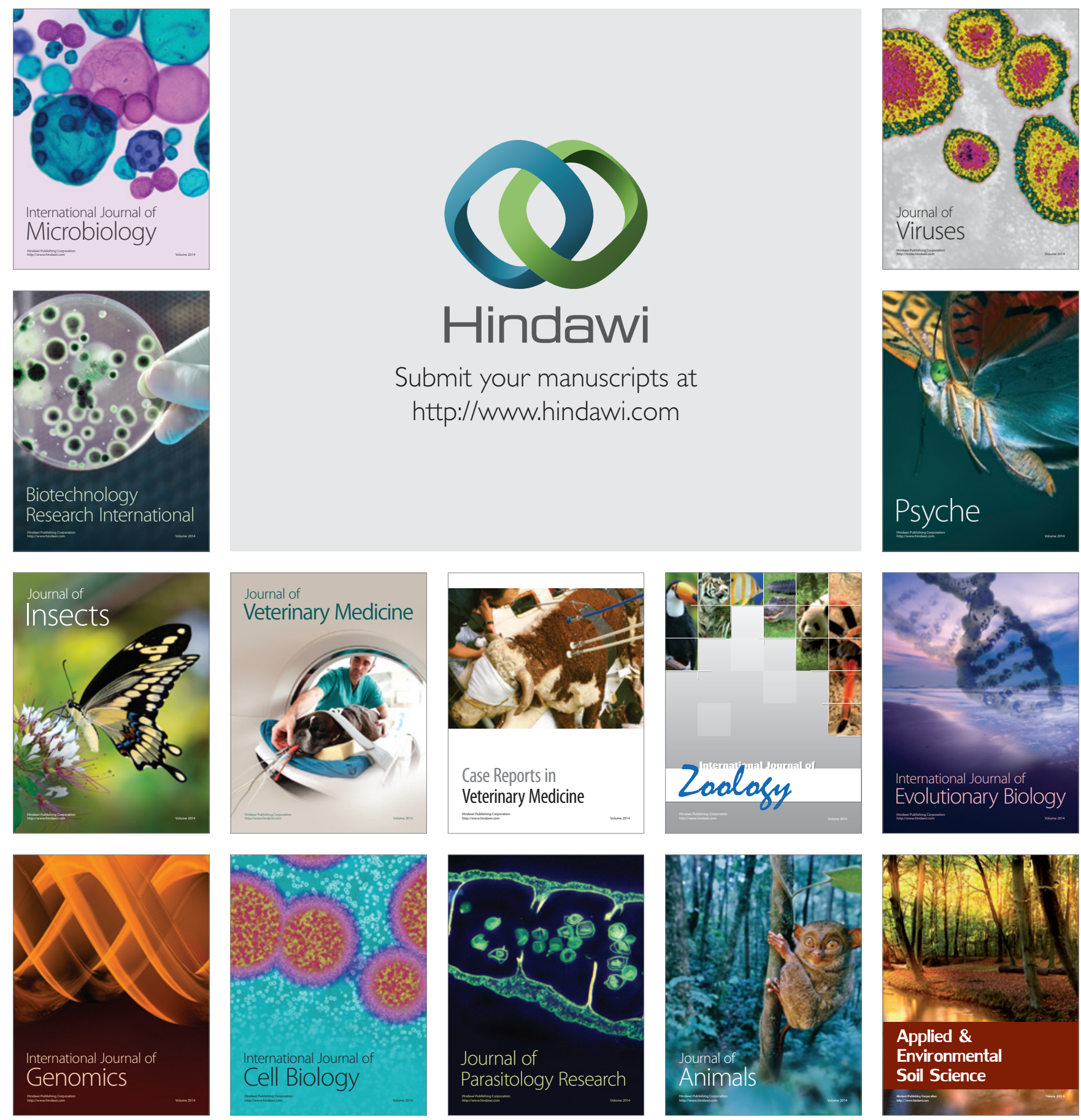